temper their fishing with a knowledge of surface geology, for it is the limestone loughs which hold the fastest growing and largest trout in Ireland.

\section{TUMOUR VIRUSES}

\section{Activating Virogenes}

from our Cell Biology Correspondent

THE hypothesis that the genomes of the normal cells of vertebrates contain genetic information required to specify RNA tumour viruses has, since it was first mooted by Bentvelzen (1968) and Payne and Chubb (1968) and then Huebner and Todaro in their eponymous oncogenes and virogenes article, dominated the thinking of many if not all tumour virologists. The idea is deceptively simple; if all animal cells vertically inherit oncogenes, the transformation of any particular cell to malignancy depends on whether its oncogenes are switched on. Likewise, the liberation of an RNA tumour virus from the cell depends on whether its virogenes are also expressed.

This hypothesis can, of course, account for the transformation of any and every cell by any and every carcinogen and the fact that most cancer cells do not seem to release an RNA tumour virus is simply explained away by saying such cells have active oncogenes but inactive virogenes. Indeed the chief problem with the hypothesis has always been that it is seemingly impossible to test, but that has not prevented quite compelling evidence in its favour being collected.

During the past five years, for example, it has been shown quite unequivocally that normal chick cells may specify an antigen indistinguishable from the group specific antigen of the avian tumour viruses and such $\mathrm{gs}^{+}$ chick cells also contain a helper factor which complements and allows to replicate defective Bryan high titre Rous sarcoma virus, RSV(O). Last year Vogt and Friis found that several $\mathrm{gs}^{+}$ chick cells spontaneously release a virus called RAV.O, which by a variety of biophysical and biochemical criteria can be classified with the avian leukosis viruses. It seemed therefore that $\mathrm{gs}^{+}$ cells do indeed inherit the potential to specify and release an avian leukosis type virus as predicted by the oncogene hypothesis. But what about gs $^{-}$cells which do not carry the gs antigenicity: do they also have the genetic potential to specify a leukosis virus? Weiss, Friis, Katz and Vogt (Virology, 46, 920 ; 1971) have now shown that they do. Using a variety of chemical carcinogens and mutagens and ionizing radiations Weiss and his colleagues have induced both gs $^{+}$and gs $^{-}$cells to start to release a virus which very closely resembles, and may be identical to, the spontaneously released virus RAV.O. The virus can be induced not only from cells of domestic chicks but also from the red jungle fowl Gallus gallus.

The induced virus contains reverse transcriptase, a single stranded $70 \mathrm{~S}$ RNA genome and virion polypeptides similar to those of the avian sarcoma leukosis viruses. The induced virus can also act as helper for the replication of the defective Bryan high titre Rous sarcoma virus and in the taxonomic sense at least it must be classified with the avian leukosis viruses. Whether it can actually cause leukosis in chicks remains to be established; if it cannot cause leukosis or transform cells in culture the possibility of rendering this virus oncogenic by, for example, repeatedly passaging it through chick cells transformed to malignancy by chemical carcinogens will have to be investigated.

What is true of chicks should, of course, be true of other vertebrates if the oncogene hypothesis is correct, and Rowe et al. (ibid., 46, $866 ; 1971$ ) have now shown that apparently virus free cells of clones of AKR mouse embryo cells, on exposure to physical carcinogens, or, significantly, on transformation by SV40, are induced to liberate a murine leukaemia virus identical to that which can cause leukaemia in AKR mice. The obvious conclusion seems to be, therefore, that both chicks and mice do indeed inherit the genetic potential to specify an RNA virus of the C-type sarcoma leukaemia virus type, and that expression of this potential can be induced by carcinogenic agents, albeit at a low frequency. Those tumour virologists wedded to the oncogene hypothesis now have powerful ammunition with which to assail the sceptics.

\section{CANCER RESEARCH \\ Oncogenic Viruses}

from a Correspondent

A SINGLE cause for all cancers has always been an attractive postulate, though heavily criticized by clinicians who see patients with many and apparently different malignant diseases. Those researchers who work with chemical carcinogens have tended to think that their favourite compounds, for example, polycyclic hydrocarbons or nitrosamines, are the most likely candidates for the chief environmental cause of cancer. Virologists have during the past decade begun to think that their pets are the most significant aetiological factor. At the British Museum (Natural History) on February 14, the sixth meeting in the series on the principles of clinical and experimental oncology gave some hint that the virologists have a point.

Dr P. M. Biggs (Houghton Poultry
Research Station) said he wished to make it clear that there seemed to be nothing special about oncogenic DNA viruses in either shape, size, encapsulation, molecular weight of nucleic acid or base ratios. They can be found among most of the recognized classes of viruses. There is the clear suggestion in such a statement that there may prove to be far more viruses which can have oncogenic effects than have so far been detected--there is even the possibility that all viruses can be oncogenetic under appropriate circumstances. Aside from the taxonomic categorization, three types of virus can be distinguished; those which have been shown to cause tumours in experimental animals, for example, polyoma and SV40; those which lead to benign tumours naturally, for example, certain pox and papilloma viruses; and those which lead to malignancies in nature, for example, Marek's disease virus in poultry.

Inferential evidence suggests that one viral genetic coding unit only is required to bring about the transformation of a cell to a malignant precursor cell. This in itself provides some validation for the notion of the oncogene. Dr Biggs pointed out that in many instances the virus was involved in a non-productive infection in rapidly growing tumour cells but that this could not be taken as indicative of lack of the viral genome. In Marek's disease, for example, a lymphoproliferative neoplasia, it is extremely difficult to identify the causative virus anywhere except in the feather follicle epithelium. In practice, vacuum cleaned debris from infected birds has been shown to contain many virus particles.

Professor W. F. H. Jarrett (University of Glasgow) spoke of the oncornaviruses particularly in fowls, mice and cats, in which they can lead to leukaemia or sarcoma. All these viruses, independent of their species of origin, have an identical physical structure and method of replication. The manner in which they succeed in instructing their host cells to synthesize replicas by production of an RNADNA polymerase is well known, at least in principle. Again the evidence shows that the cells in which malignant transformation occurs are often not those in which virus is produced. Professor Jarrett indicated that the strongest evidence against the extreme form of the ubiquitous oncogene theory, which requires vertical transmission from generation to generation of genetic information, perhaps in the form of an RNA viral genome or its DNA replica, is that horizontal transmission can be made to occur and it does lead to malignancy. In these circumstances Occam's razor excises the oncogene. 